\title{
Causes of Weak-localization of Electrons on Liquid Helium
}

\author{
Annie Wakata, Paul Leiderer, and Jürgen Klier \\ Department of Physics, University of Konstanz, D-78457 Konstanz, Germany \\ E-mail: juergen.klier@uni-konstanz.de
}

We have investigated the dephasing processes in a weak localization (WL) experiment of a two-dimensional electron system on liquid helium. From low-field magnetoconductivity measurements we can separate the damping of $W L$ on the dephasing of electrons due to electron-electron interaction and the motion of the helium vapour atoms. We observe an intermediate regime where both damping mechanisms are of comparable importance and determine the cross-over from one dominant regime to the other.

PACS Numbers: 73.20.-r; 73.20.Fz; 73.20.Hb; 73.50.Dn.

\section{INTRODUCTION}

Weak localization (WL) is a quantum effect caused by the coherence among multiple elastic-scattering paths of a conduction electron. This coherence leads to an enhanced backscattering probability and an increase in the resistivity over the classical Drude model. WL theory is now on firm ground and has been verified in both degenerate ${ }^{1-4}$ and more recently in non-degenerate electron systems. The first experiment in non-degenerate systems explored electrons on a solid hydrogen surface ${ }^{5}$ while the most recent experiment ${ }^{6}$ was carried out, like in our system, on electrons confined to two dimensions (2D) above a liquid helium surface. In the system presented in this paper WL results from quasi-elastic scattering from slowly moving helium vapour atoms.

The experiments are performed in a temperature regime $1.9<T<2.2 \mathrm{~K}$ were scattering from quantized surface waves can be neglected. The most fundamental property of the electron is the time over which the phase coherence is maintained in its wave function. In the standard theory of $\mathrm{WL}$, it is assumed that the scattering centres are at fixed positions in space, but it has also been shown ${ }^{7}$ that WL can occur in the case of 
moving scatterers. It is well understood that the coupling of the electron system to an environment of moving scatterers will cause a reduction in the constructive interferences of all possible paths due to changes induced in the wave function of the electron by this environment. The standard approach $^{8}$ for determining the phase coherence time in $1 \mathrm{D}$ and $2 \mathrm{D}$ systems is to fit weak localization theory to the measured change in resistance as a function of an applied magnetic field.

Decoherence can be caused by the motion of the helium vapour atoms and by the electron-electron interaction. The motion of the scattering sites leads to an unusual damping mechanism for quantum effects. In condensed matter electron systems (metallic films and electron gas in semiconductors) the scatterers are usually quasi-static and the damping mechanisms lead to a simple exponential decay of the interference. In contrast to this standard behaviour damping due to the motion of helium vapour atoms leads to a cubic exponential decay: the interference contribution of the electron paths of duration $t$ is reduced by a factor $\exp \left[-\left(t / \tau_{3}\right)^{3}\right]{ }^{9-11}$ Here $\tau_{3}$ is the effective damping time due to the vapour atom motion.

In the context of quantum transport, the form of damping can be probed by measuring the magnetic field dependence of the WL correction, as first pointed out by Afonin et al. ${ }^{9}$ Karakurt et al. ${ }^{6}$ reported recently an experimental investigation of the damping of WL of electrons on liquid helium and found empirically that the electron-electron interaction produces a simple exponential damping, though a theory of damping due to the electron-electron interaction is lacking for non-degenerate electron systems. In that work, ${ }^{6}$ the contributions of electron-electron interaction and vapour-atom motion were separated, but direct evidence that the interaction of the electrons with the moving helium vapour atoms leads to a cubic exponential damping was not given. In a comparable system, Adams and Paalanen, ${ }^{5}$ reported a simple exponential damping in a situation where damping by helium gas atoms was relevant. One explanation could be that the expression for the cubic exponential damping was cumbersome to apply and would lead to small shifts in the measured values of the dephasing times, since the expressions used were of first order in perturbation. Recently, Herman et al. ${ }^{12}$ found that the exponential decay gives a satisfactory fit even when the damping is dominated by the motion of the helium atoms.

For WL of the electrons one has to consider two damping time scales: a simple exponential decay time $\tau_{1}$ expected due to electron-electron interaction damping, ${ }^{12}$ and a cubic exponential damping time $\tau_{3}$ due to interaction with the moving vapour atoms. ${ }^{9-11}$ The total dephasing time 
$\tau_{\phi}$ is defined by $\tau_{\phi}^{-1}=\tau_{1}^{-1}+\tau_{3}^{-1}$. For a non-degenerate $2 \mathrm{D}$ electron fluid the longitudinal conductivity in the low-magnetic field including the WL corrections is given by, ${ }^{5,13-15}$

$$
\begin{aligned}
\sigma_{x x}= & \frac{-n e^{2}}{m\left(k_{B} T\right)^{2}} \int_{E_{c}}^{\infty} \exp \left(\frac{-E}{k_{B} T}\right) d E \\
& \times\left\{E \tau_{0}+\frac{\hbar}{2 \pi} \phi(E, B)\right\} \frac{1}{1+(\mu B)^{2}} .
\end{aligned}
$$

The first term in the curly brackets corresponds to the classical Drude conductivity which is justified in the low-field limit. The second term is the correction term arising from WL effects. Here $n$ is the electron density, $m$ is the electron mass, $E$ is the electron energy, $\mu$ is the electron mobility, $B$ is the perpendicular magnetic field, and $E_{c}=\hbar / 2 \pi \tau_{0} \ln \left(\tau_{\phi} / \tau_{0}\right)$ is the strong localization threshold below which the electrons are presumed to be strongly localized and do not contribute to the conductance. $\tau_{0}$ is the quasi-elastic scattering time. In Eq.(1), the function $\phi(E, B)$ is defined as $^{5,11}$

$$
\phi(E, B)=\int_{\tau_{0}}^{\infty} \frac{4 \pi e D B}{h} \frac{\exp \left(-t / \tau_{1}\right) \exp \left(-t^{3} / \tau_{3}^{3}\right)}{\sinh (4 \pi e D t B / h)} d t
$$

with $D=E \tau_{0} / m$ as the electron diffusion constant. Equation (2) is a more general form that describes simultaneously the simple exponential and the cubic exponential damping mechanisms present in the system. Due to the flexibility of the electron system above liquid helium, several variable parameters allow to examine the intermediate regime and to study how it crosses over from one extreme to the other. In this paper, we present detailed measurements in the three different regimes discussed above. We worked in a wide range of electron densities from $5 \times 10^{11} \mathrm{~m}^{-2}$ down to the lowest density of $2 \times 10^{10} \mathrm{~m}^{-2}$.

\section{EXPERIMENTAL METHODS}

For the experiments a Corbino geometry ${ }^{16}$ consisting of five circular electrodes located beneath the liquid helium surface was used, see inset in Fig.1. The conductivity $\sigma_{x x}$ is directly measured by applying an ac voltage $(10 \mathrm{mV}$ at $40 \mathrm{kHz})$ to electrode $\mathrm{B}_{1}$, and detecting the ac current induced to electrode $\mathrm{B}_{3}$ with a lock-in amplifier. Both measuring electrodes are separated by electrode $B_{2}$ to minimize direct coupling. The electrons are held in place by de potentials applied to electrodes $B_{1}, B_{2}, B_{3}$, and to the in-plane guard ring $G$. The density profile at the border of the electron 


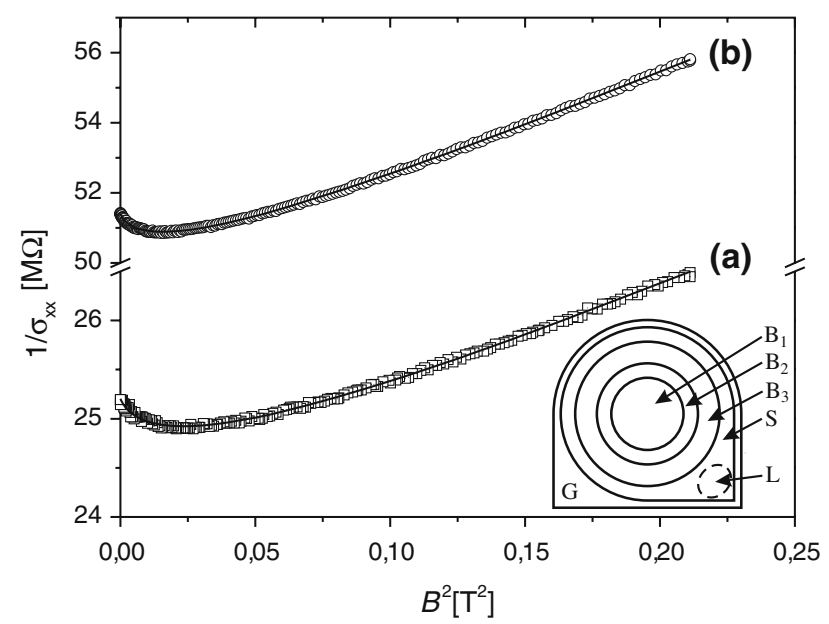

Fig. 1. Measured inverse magnetoconductivity $1 / \sigma_{x x}$ versus $B^{2}$. For (a) $n=4.09 \times 10^{11} \mathrm{~m}^{-2}$ at $T=2.15 \mathrm{~K}$ and for (b) $n=1.68 \times 10^{11} \mathrm{~m}^{-2}$ at $T=2.12 \mathrm{~K}$. The solid lines show the fits for the parameters given in Table I. We assume both kinds of damping are present and the values of $\tau_{1}$ and $\tau_{3}$ correspond to a simple exponential damping regime. Inset: Corbino arrangement. $\mathrm{G}$ is the guard ring, $\mathrm{S}$ the shaping electrode, $\mathrm{B}_{1}$ to $\mathrm{B}_{3}$ the measuring electrodes, and $\mathrm{L}$ indicates the electron loading area. The diameter of the electrodes $B_{1}$ to $B_{3}$ is about 11.0, 14.8 and $18.6 \mathrm{~mm}$, the spacing between is $0.1 \mathrm{~mm}$.

sheet is controlled with the positive potential applied to the shaping electrode S. The idea of using the shaping electrode is to have a uniform density over the measuring electrodes and avoid non-uniformity at the edges of these electrodes. The density profile above the electrodes was calculated numerically and the density uniformity above the measuring electrodes in the presence of the shaping electrode was verified. These calculations give the value of the density at saturation from the field distribution in the cell. But the experiments presented here were not performed at saturated electron density. The same set of voltages (holding voltage, shaping and guard) was used for each temperature and only the charging time was varied from one run to the other. The helium surface was charged with the electrons only once at the beginning, and not during the measurements. The measurements were then conducted with the same field distribution, same area of the electron pool, but different amount of electrons for different densities. For most results presented in this paper a guard field of $-1.5 \mathrm{kVm}^{-1}$ was used and the potential applied to the shaping electrode is always equal to the ones applied to the three inner electrodes. The thickness of the helium layer above the electrodes was about $0.5 \mathrm{~mm}$. 


\section{EXPERIMENTAL RESULTS AND ANALYSIS}

Shown in Fig. 1(a) is the inverse magnetoconductivity, $1 / \sigma_{x x}$, of the 2D electron gas on liquid helium with a density $n=4.09 \times 10^{11} \mathrm{~m}^{-2}$ at $T=2.15 \mathrm{~K}$. The uncertainty in density is less than $0.005 \times 10^{11} \mathrm{~m}^{-2}$ for all measured values. Small temperature drifts may occur during the measurements, but they were negligible (less than $1 \mathrm{mK}$ in 10 hours) and each experimental curve was reproduced at least four times. Note the negative derivative of $1 / \sigma_{x x}$ at low fields due to WL effects and the positive $B^{2}$ dependence at higher fields representing the Drude resistivity. The normal holding field applied to the measuring electrodes is $880 \mathrm{Vm}^{-1}$, that is four times lower than the saturated field $E_{s}=n e / 2 \epsilon_{0}$. The best fit, using Eqs. (1) and (2), is also shown with the corresponding parameters $\tau_{0}=4.30 \mathrm{ps}, \tau_{1}=19.78 \mathrm{ps}$, and $\tau_{3}=890 \mathrm{ps}$. So, here the simple exponential damping, due to electron-electron interaction, is the dominating damping mechanism, which can be explained by the relatively high-electron density. The quality of the fit is determined by a parameter $\chi^{2}$ defined as $\chi^{2}=$ $\sum_{i}\left[\left(\rho_{m}^{\left(B_{i}\right)}-\rho_{x x}^{\left(B_{i}\right)}\right) / \rho_{x x}^{\left(B_{i}\right)}\right]^{2} . i$ represents each measured point, $B_{i}$ the corresponding magnetic field, $\rho_{m}^{\left(B_{i}\right)}$ the measured resistivity at $B_{i}$, and $\rho_{x x}$ the resistivity calculated from Eq. (1). For the fits presented here $\chi^{2}$ is in the range $6 \times 10^{-6}$ to $3 \times 10^{-5}$.

For the data shown in Fig. 2(a) the field distribution in the cell and the temperature are the same as in Fig. 1(a) but now the density is two times smaller, i.e. $n=2.08 \times 10^{11} \mathrm{~m}^{-2}$. The best fit is found for $\tau_{0}=4.00 \mathrm{ps}$ $\tau_{1}=24.00 \mathrm{ps}$ and $\tau_{3}=86.77 \mathrm{ps}$. When one reaches $n=1.72 \times 10^{11} \mathrm{~m}^{-2}$ (with the same field distribution and at the same temperature), the characteristic times are $\tau_{0}=4.12 \mathrm{ps}, \tau_{1}=82.43 \mathrm{ps}$ and $\tau_{3}=23.92 \mathrm{ps}$, see Fig. 2(b). So for these densities both damping mechanisms are present and the cubic exponential damping becomes important. The dependence of $1 / \sigma_{x x}$ versus $B^{2}$ (for $B \rightarrow 0$ ) cuts off more sharply than a simple exponential damping would cause. We see that as the density is decreased, the motion of helium atoms becomes more important, and it finally dominates the damping factor. To clearly illustrate this, we did measurement at a lower density, and for evident reasons of the signal quality, we worked at a slightly lower temperature. The quality of the signal depends on temperature and density of the electrons. At high $T$ and low $n$, the ratio of signal over noise is not so good and hence an investigation of the system at low densities requires a somewhat lower $T$.

In Fig. 1(b), the electron density is $1.68 \times 10^{11} \mathrm{~m}^{-2}$ and the normal holding field is $880 \mathrm{Vm}^{-1}$. The temperature is $2.12 \mathrm{~K}$. The best fitting parameters are $\tau_{0}=4.90 \mathrm{ps}, \tau_{1}=22.05 \mathrm{ps}$ and $\tau_{3}=474.1 \mathrm{ps}$. Here the helium 


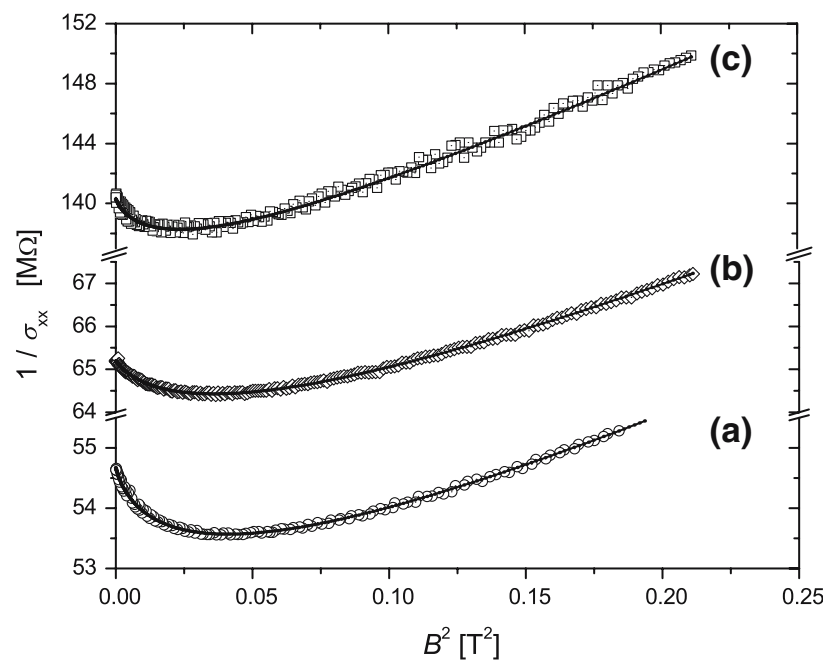

Fig. 2. Measured values of $1 / \sigma_{x x}$ versus $B^{2}$. For (a) $n=2.08 \times 10^{11} \mathrm{~m}^{-2}$ and for (b) $n=1.72 \times$ $10^{11} \mathrm{~m}^{-2}$, both at $T=2.15 \mathrm{~K}$. For (c) $n=0.64 \times 10^{11} \mathrm{~m}^{-2}$ at $T=2.12 \mathrm{~K}$. The solid lines show the fits with the parameters given in Table I. Curve (a) corresponds to an intermediate regime with $\tau_{1}$ damping more important, in curve (b) $\tau_{3}$ damping is more important, and in curve (c) both damping mechanisms have comparable importance.

vapour pressure is smaller than previously and the damping process is essentially due to electron-electron interaction.

For the same field distribution and lower density, $n=0.64 \times 10^{11} \mathrm{~m}^{-2}$, the characteristic times are $\tau_{0}=4.90 \mathrm{ps}, \tau_{1}=44.10 \mathrm{ps}$ and $\tau_{3}=40.35 \mathrm{ps}$. The state of the system is in an intermediate regime with both damping mechanisms, and both are of the same importance, see Fig. 2(c).

We are not working at saturated electron densities. ${ }^{17}$ The first estimation of both density and mobility is given from the slope of $1 / \sigma_{x x}$ with $B^{2}$ at higher field for the mobility, and $1 / \sigma_{x x}$ at zero field, in the absence of WL, for the density. $n$ and $\tau_{0}$ are then adjusted to get the best fit in order to determine the dephasing times (the value of the quasielastic scattering time $\tau_{0}$ is derived from the mobility, i.e. $\mu=e \tau_{0} / m$ ). This can lead to small shifts on these two quantities (lower than $2 \%$ ). $\tau_{1}$ and $\tau_{3}$ are varied over a wide range until we get the best fit that is given by the minimum of $\chi^{2}$. The total dephasing time is set in the program as a real number $r$ multiplied by $\tau_{0}$ and $\tau_{1}$ is set as a number $r_{1}$ times $\tau_{0}\left(r_{1}\right.$ is necessarily higher or equal to $r$, according to the definition of the total dephasing time). The physical value of $r$ is usually a real number between 3 and 5 in the conditions (temperature) in which we are working, it represents the number of scattering of one electron before it looses its coherence. $r_{1}$ is varied over 
a wide range, starting from $r$ (assuming that there is only simple exponential decay) and increased to higher values (slowly introducing the cubic exponential damping) until the best fit is found. The error on $\tau_{1}$ and $\tau_{3}$ is about $0.1 \%$. For the measurements at $2.15 \mathrm{~K}$ we find a mean value of $4.12 \mathrm{ps}$ for $\tau_{0}$ with a standard deviation of $0.01 \mathrm{ps}$. The $1 / \sigma_{x x}$ line shapes at $2.12 \mathrm{~K}$ are well fitted with the same value of $\tau_{0}$ and $\tau_{\phi}$.

In Ref. 6, the behaviour of the dephasing rates for both the vertical and horizontal motion of the vapour atoms is studied. The idea is 6,15 that the effective Bohr radius of the electron pool being finite, the scattering atom may not be present for both the forward and return paths. The weight of the interference contribution is thereby reduced by the vertical motion. In contrast, ${ }^{9,10}$ the horizontal motion changes the lengths of the paths introducing a random relative phase between the interfering waves, thereby washing out their interference.

We did other measurements with a slightly different field distribution. Figure 3 shows by the solid line the best fit to $1 / \sigma_{x x}$ for $n=0.34 \times$ $10^{11} \mathrm{~m}^{-2}$ at $T=2.12 \mathrm{~K}$. For this sample, the holding field was $820 \mathrm{Vm}^{-1}$ and the guard field was $-2 \mathrm{kVm}^{-1}$. On one hand the increase of the

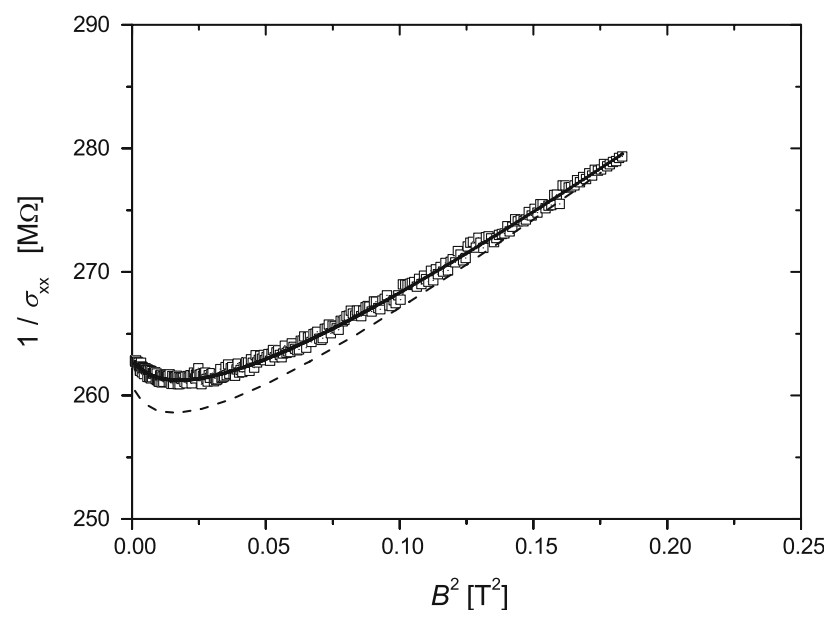

Fig. 3. Measured values of $1 / \sigma_{x x}$ versus $B^{2} ; n=0.34 \times 10^{11} \mathrm{~m}^{-2}$ at $T=2.12 \mathrm{~K}$. At this low electron density the extreme regime of damping dominated due to the motion of vapour atoms is reached. The corresponding fit is shown as solid line with the parameters given in Table I. The dashed line represents a fit assuming the simple exponential damping dominating in the data. For that $\tau_{0}$ is taken to be $4.90 \mathrm{ps}$, as measured for $T=2.12 \mathrm{~K}$, and by minimizing $\chi^{2}$ we get $\tau_{1}=21.56 \mathrm{ps}$ while $\tau_{3} \rightarrow \infty$. We also fitted the data by varying $\tau_{0}$ and $\tau_{1}$ simultaneously, and found as best fit (not shown) $\tau_{0}=4.6 \mathrm{ps}$ and $\tau_{1}=19.32 \mathrm{ps}$ while $\tau_{3} \rightarrow \infty$. So, obviously these calculated values for $1 / \sigma_{x x}$ do not fit the data. 


\section{TABLE I}

Parameters used for the best fits of $1 / \sigma_{x x}$ vs $B^{2}$ (sorted by density): electron density $n$, dephasing time $\tau_{1}$ (due to electron-electron interaction), dephasing time $\tau_{3}$ (due to the motion of vapour-atoms), and the quasielastic scattering time $\tau_{0}$. The uncertainty for $\tau_{1}$ and $\tau_{3}$ for the data in Fig. 3 is about $1 \%$, in all other cases it is much less than $1 \%$.

\begin{tabular}{lccccc}
\hline Fig. & $n\left[10^{11} \mathrm{~m}^{-2}\right]$ & $T[\mathrm{~K}]$ & $\tau_{1}[\mathrm{ps}]$ & $\tau_{3}[\mathrm{ps}]$ & $\tau_{0}[\mathrm{ps}]$ \\
\hline 1a & 4.09 & 2.15 & 19.78 & 890.1 & 4.30 \\
2a & 2.08 & 2.15 & 24.00 & 86.77 & 4.00 \\
$2 \mathrm{~b}$ & 1.72 & 2.15 & 82.43 & 23.92 & 4.12 \\
$1 \mathrm{~b}$ & 1.68 & 2.12 & 22.05 & 474.1 & 4.90 \\
4 & 1.22 & 2.12 & 24.50 & 150.5 & 4.90 \\
4 & 0.69 & 2.12 & 29.40 & 74.36 & 4.90 \\
$2 \mathrm{c}$ & 0.64 & 2.12 & 44.10 & 40.35 & 4.90 \\
3 & 0.34 & 2.12 & 2450 & 21.75 & 4.90 \\
\hline
\end{tabular}

thickness of the electron layer should increase the dephasing time due to the vertical motion of the helium vapour atoms. While the change in the guard voltage would increase damping due to the horizontal motion of the helium atoms, it could also increase the electron confinement and so reduce their mobility. The corresponding dephasing times are $\tau_{0}=4.90 \mathrm{ps,}$ $\tau_{1}=2450 \mathrm{ps}$ and $\tau_{3}=21.75 \mathrm{ps}$. For this low-electron density the damping mechanism follows the cubic exponential law, i.e. governed by collisions with helium vapour atoms. We think the small changes in the holding and guard voltages have negligible effects, since $\tau_{0}$ is the same as before, and the density profile above the measuring electrodes is not affected due to the presence of the shaping electrode. The relative change of the width of the electronic wave function is about $10^{-4}$, hence induces a negligible change in the damping due to the vertical motion of the electrons. The different samples are thus comparable and the only parameters changed in the system are the electron density and the temperature. A summary is given in Table I.

\section{DISCUSSION OF RESULTS}

The dependence of $\tau_{1}$ and $\tau_{3}$ with electron density is shown in Fig. 4 for measurements at $2.12 \mathrm{~K}$. The holding field was about $800 \mathrm{Vm}^{-1}$, except for the measurement at $n=0.68 \times 10^{11} \mathrm{~m}^{-2}$ for which a value of $700 \mathrm{Vm}^{-1}$ was applied. Qualitatively we observe the same behaviour for $\tau_{1}$ and $\tau_{3}$ at $2.15 \mathrm{~K}$, but the intermediate regime is shifted to slightly higher densities, as expected. The density of the helium vapour is higher at higher $T$. 


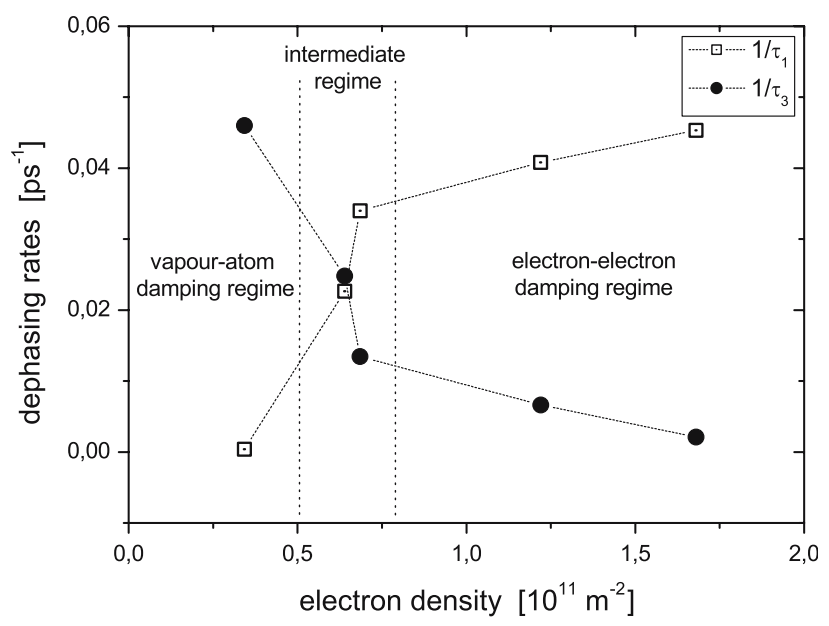

Fig. 4. Inverse dephasing times $\tau_{1}$ and $\tau_{3}$ vs electron density at $T=2.12 \mathrm{~K}$. One observes the cross-over from one regime to the other as the density varies. The lines between the data points are just a guide to the eye.

Therefore, the influence of the vapour atoms is greater than at $2.12 \mathrm{~K}$, so that dephasing due to the motion of the atoms occurs at higher electron densities. In Fig. 4, one observes a kind of symmetry between the values of $\tau_{1}$ and $\tau_{3}$. This is due to the relation between $\tau_{1}$ and $\tau_{3}$ as far as $\tau_{\phi}$ is concerned, and the fact that the measured total dephasing time is the same, i.e. $\tau_{\phi}=21.07 \mathrm{ps}$ at $2.12 \mathrm{~K}$; except for $n=0.68 \times 10^{11} \mathrm{~m}^{-2}$ for which $\tau_{\phi}=21.56 \mathrm{ps}$. The uncertainty in $\tau_{\phi}$ is around $0.01 \mathrm{ps}$. For this density the holding field is different, as said above. This could indicate that the field distribution in the cell affects the total dephasing time. In Refs. 6, 12 , changes in the mobility as a function of the holding field are reported, as well as variations in $\tau_{1}$ and $\tau_{3}$ at fixed density and temperature with the holding voltage.

For each data set, Figs. 1-3, we tried three fits assuming linear, cubic and intermediate regimes, and looked for the parameters that give the best fit for each regime. When the best fit is for example in one regime, the fits assuming the two other regimes are good either at low $B$ or at high $B$, and the lowest value of $\chi^{2}$ for these is at least 20 times higher than its value for the best fit. The fact that the density is adjusted during the fitting process makes that we can not get two good fits with different values of $\tau_{1}$ and $\tau_{3}$. Though it is not specified in Ref. 12 the authors always assume saturated electron densities in their measurements, ${ }^{18}$ so they can calculate the density independently and do not use it as a fitting 
parameter. That could explain why they can find two good fits with different values of $\tau_{1}$ and $\tau_{3}$. One in the $\tau_{1}$ regime and one in the intermediate regime dominated by $\tau_{3}$.

The absolute values of $\chi^{2}$ alone can not be compared to the ones in Ref. 12, since it depends on the number of fitted points and on the dispersion of the measured points. The values of $\chi^{2}$ given here were calculated either for a set of 50 points or for more than 200 points (all the measured points). These values cannot be compared to those published before Ref.12 since the number of fitted points was not specified. Some of the values given for $\tau_{1}$ may seem rather unphysical (e.g. $\tau_{1}=2450 \mathrm{ps}$ ), this is caused by the fact that setting $r_{1}$ to infinity causes problems to the fitting programme. The ratio of $\tau_{1}$ and $\tau_{3}$ to the total dephasing time is more relevant. In the particular example quoted here, $\tau_{3}$ is nearly equal to the total dephasing time. It just means that the contribution of $\tau_{1}$ is highly negligible compared to $\tau_{3}$, and that the principal damping mechanism is the cubic exponential relaxation.

From our measurements we could not verify the assumption ${ }^{6}$ that the dephasing mechanism due to the motion of helium atoms is independent of the electron density. In Ref. 6 a fixed value of 24 ps is measured for the dephasing time due to the motion of the helium vapour atoms over a density range from $7 \times 10^{9} \mathrm{~m}^{-2}$ to $4 \times 10^{11} \mathrm{~m}^{-2}$ at $T=1.96 \mathrm{~K}$. Since the theory of dephasing due to the electron-electron interaction is still not complete for a non-degenerate electron gas, it is difficult to draw a conclusion and do comparison with data where the density, the holding fields and the guard fields were simultaneously varied. The influence of the field distribution is also questionable. A support for this assumption comes from measurements available in comparable systems $\mathrm{s}^{5,6}$ in which the authors reported the best fits for simple exponential damping in situations where the dephasing was theoretically dominated by scattering with moving helium atoms.

Although theory predicted the existence of the cubic exponential damping due to the motion of the scattering sites, it took some time to introduce it in the WL correction. In Ref. 6, the function used to fit the data is the one that is usually used for WL in degenerate electron systems; so a simple exponential decay within a time $\tau_{\phi}$ was considered. The total dephasing time was defined as $\tau_{\phi}^{-1}=\tau_{e e}^{-1}+\tau_{A}^{-1}$ (see Eq. (2) in Ref. 6; to compare with our notations, $\tau_{e e}$ equals $\tau_{1}$ and $\tau_{A}$ stands for $\tau_{3}$ ). The data published in Ref. 6 were reanalyzed in Ref. 12 using a fitting function that took into account both kinds of damping mechanisms. The theoretical analysis used in the present paper is based on the results in Ref. 12. Instead of casting doubt on previous experimental work, our analysis is 
building on it. To compare our results to these earlier experimental ones, we find nearly the same values for $\tau_{0}$ and $\tau_{\phi}$ when the temperature and the densities are comparable. The main difference from the previous works is that we performed experiments in a wide range of non-saturated electron densities with the same field distribution in the cell, which allows the system to reach different regimes related to the contribution of the two dephasing processes.

Another problem of the theory ${ }^{11}$ is the statement that the total dephasing rate is defined as $\tau_{\phi}^{-1}=\tau_{1}^{-1}+\tau_{3}^{-1}$. This equation can not apply correctly since $\tau_{3}$ does not follow the simple exponential decay as $\tau_{1}$. So, a better theoretical understanding of the system 'weak localization' is necessary.

\section{CONCLUSIONS}

In summary, we have investigated the damping of WL of electrons on liquid helium. We observed the presence of both predicted damping mechanisms: simple exponential damping due to electron-electron interaction and cubic exponential damping due to helium vapour atom motion. By changing only the electron density the two extreme regimes are presented. In the intermediate density regime both mechanisms are comparable. To our knowledge, this is the first observation of a cross-over from the simple exponential decay to the cubic exponential damping in such a system.

\section{ACKNOWLEDGEMENTS}

We thank A.J. Dahm and I. Karakurt for helpful discussions. One of us (A.W.) is grateful to the Alexander von Humboldt Foundation for a grant. This activity was supported by the DFG, Forschergruppe 'Quantengase', and the EU-RTN 'Surface electrons on mesoscopic structures'.

\section{REFERENCES}

1. G. Bergmann, Phys. Rep. 107, 1 (1984).

2. P. A. Lee and T. V. Ramakrishnan, Rev. Mod. Phys. 57, 287 (1985).

3. S. Chakravarty and A. Schmid, Phys. Rep. 140, 193 (1986).

4. D. J. Bishop, R. C. Dynes, and D. C. Tsui, Phys. Rev. B 26, 773 (1986).

5. P. W. Adams and M. A. Paalanen, Phys. Rev. Lett. 58, 2106 (1987). [ibid, Phys. Rev. B 39, 4733 (1989); P.W. Adams, Phys. Rev. Lett. 65, 3333 (1990)].

6. I. Karakurt, D. Herman, H. Mathur, and A. J. Dahm, Phys. Rev. Lett. 85, 1072 (2000).

7. V. V. Afonin, Yu. M. Galperin, and V. L. Gurevich, Sov. Phys. - JETP 61, 1130 (1985).

8. B. L. Altshuler and A. G. Aronov, in Electron-Electron Interactions in Disordered Systems, A.L. Efros and M. Pollak (eds.), Elsevier, Amsterdam (1985).

9. V. V. Afonin, Yu. M. Galperin, V. L. Gurevich, and A. Schmid, Phys. Rev. A 36, 5729 (1987). 
10. M. J. Stephen, Phys. Rev. B 36, 5663 (1987).

11. D. Herman, H. Mathur, and A.J. Dahm, Phys. Rev. B 63, 115418 (2001).

12. D. Herman, I. Karakurt, H. Mathur, and A.J. Dahm, Phys. Rev. B 68, 033402 (2003).

13. B. L. Altshuler, D. Khmel'nitzkii, A. I. Larkin, and P. A. Lee, Phys. Rev. B 22, 5142 (1980).

14. V. V. Afonin, Yu. M. Galperin, and V. L. Gurevich, Phys. Rev. B 40, 2598 (1989).

15. M. J. Stephen, Phys. Rev. B 40, 2600 (1989).

16. O. M. Corbino, Phys. Zeit. 12, 561 (1911).

17. A saturated density is difficult to reach practically, and one cannot determine the density only from the applied fields.

18. I. Karakurt, Private Communication. 